\title{
Local Influence for Generalized Linear Models with Missing Covariates
}

\author{
Xiaoyan Shi, Hongtu Zhu, and Joseph G. Ibrahim * \\ Department of Biostatistics, University of North Carolina at Chapel Hill, Chapel Hill, North Carolina \\ 27599, U.S.A.
}

\section{Summary}

In the analysis of missing data, sensitivity analyses are commonly used to check the sensitivity of the parameters of interest with respect to the missing data mechanism and other distributional and modeling assumptions. In this article, we formally develop a general local influence method to carry out sensitivity analyses of minor perturbations to generalized linear models in the presence of missing covariate data. We examine two types of perturbation schemes (the single-case and global perturbation schemes) for perturbing various assumptions in this setting. We show that the metric tensor of a perturbation manifold provides useful information for selecting an appropriate perturbation. We also develop several local influence measures to identify influential points and test model misspecification. Simulation studies are conducted to evaluate our methods, and real datasets are analyzed to illustrate the use of our local influence measures.

\section{Keywords}

Influence measure; Local influence; Missing covariates; Perturbation manifold; Perturbation scheme

\section{Introduction}

\begin{abstract}
Missing data are common in various settings, including surveys, clinical trials, and longitudinal studies. Methods for handling missing data strongly depend on the mechanism that generated the missing values as well as the distributional and modeling assumptions at various stages. Therefore, the resulting estimates and tests may be sensitive to these assumptions. For this reason, sensitivity analyses are commonly used to check the sensitivity of the parameter estimates of interest with respect to the model assumptions. Sensitivity analyses are often carried out in two consecutive steps: selection of perturbation schemes to various model assumptions and use of influence measures to quantify the effects of those perturbations. Some literature on sensitivity analysis for missing data problems includes Copas and $\mathrm{Li}$ (1997); Copas and Eguchi (2005); Troxel (1998); Jansen et al. (2003); Van Steen, Molenberghs, and Thijs (2001); Verbeke et al. (2001); Hens et al. (2005); Jansen et al. (2006); and Troxel, Ma, and Heitjan (2004). For instance, Copas and Eguchi (2005) proposed a general formulation for assessing the bias of maximum likelihood estimates due to incomplete data in the presence of small model uncertainty. Verbeke et al. (2001), Hens et al. (2005), and Jansen et al. (2006) developed local influence methods for assessing nonrandom dropout in incomplete longitudinal data.
\end{abstract}

\footnotetext{
(c) 2009, The International Biometric Society
}

*ibrahim@bios.unc.edu. 
Cook (1986) proposed a general approach for assessing the local influence of a minor perturbation to a statistical model, which has been applied to many types of models, such as mixed models (Beckman, Nachtsheim, and Cook, 1987), generalized linear models (GLMs; Thomas and Cook, 1989), among others. Zhu and Lee (2001) extended Cook's approach for assessing local influence in a minor perturbation of statistical models for latent variable models. Recently, Zhu et al. (2007) developed a perturbation manifold to select an appropriate perturbation for statistical models without missing data, which is central to the development of the local influence approach proposed here.

The aim of this article is to systematically investigate Cook's (1986) local influence methods for GLMs with missing at random (MAR) covariates as well as not missing at random (NMAR) covariates, often referred to as nonignorable missing covariates. Our local influence method provides a general framework for carrying out sensitivity analyses for missing data problems, compared to the existing literature (Van Steen et al., 2001; Troxel et al., 2004; Copas and Eguchi, 2005; Hens et al., 2005; Jansen et al., 2006). We examine two types of perturbation schemes for perturbing various modeling assumptions and individual observations. We also develop a methodology for selecting appropriate perturbation schemes. We examine two objective functions, including the maximum likelihood estimate and the likelihood ratio statistic, and then we develop influence measures based on these functions to assess appropriate perturbation schemes.

To motivate the proposed methodology, we consider a quality-of-life dataset and a liver cancer dataset. The quality-of-life study of the International Breast Cancer Study Group compares several chemotherapies in premenopausal women with breast cancer. These women were randomly assigned in a $2 \times 2$ factorial design to receive tamoxifen either alone or with oral cyclophosphamide, intravenous methotrexate, and flourouracil in three early cycles, three delayed cycles, or both early and delayed cycles. For ease of exposition, the four treatment arms are labeled A, B, C, and D. The response variable is the logarithm of the survival time. The dataset has 404 observations and the covariates are: physical ability; mood; indicator for treatment A (yes, no); indicator for treatment B (yes, no); indicator for treatment C (yes, no); age (in years); and language (English, otherwise). Among these seven covariates, physical ability and mood have $13 \%$ and $31 \%$ missingness percentages, respectively, and the remaining covariates are fully observed. The liver cancer dataset has 191 patients from two Eastern Cooperative Oncology Group clinical trials (Ibrahim, Chen, and Lipsitz, 1999). Previous analyses of these data focused on characterizing how the number of cancerous liver nodes (response) when entering the trials was predicted by six other baseline characteristics: time since diagnosis of the disease (in weeks); two biochemical markers (alpha-fetoprotein and antihepatitis B antigen, each classified as normal or abnormal); associated jaundice (yes, no); body mass index (weight in kilograms divided by the square of height in meters); and age (in years). Among these six covariates, three have missing data and the remaining covariates are completely observed. The three with missing data, which are time since diagnosis of the disease, alpha-fetoprotein, and anti-hepatitis B antigen, have $8.9 \%, 5.8 \%$, and $18.3 \%$ missingness percentages, yielding a total missingness percentage of $29 \%$. Here, it is of interest to carry out local influence methods to possibly detect influential cases and to carry out sensitivity analyses on the modeling assumptions. For instance, using our new methodology, we detected that cases $10,15,65$, and 160 in the liver cancer data have abnormally large response values, and case 131 has an extreme covariate value in time since diagnosis compared to the rest of the cases (Table 1). More details regarding these two real datasets are given in Section 5.

The article is organized as follows. In Section 2, we review the model development for GLMs with missing covariates. In Section 3, we systematically develop local influence measures for assessing small perturbations to modeling assumptions in GLMs with missing covariates. We 
present several simulation studies in Section 4, and analyze two real datasets in Section 5. We conclude the article with some final remarks in Section 6.

\section{Model and Notation}

Suppose that we have complete data $D_{c}=\left\{\mathbf{d}_{i}=\left(\mathbf{x}_{i}, \mathbf{z}_{i}, \mathbf{r}_{i}, y_{i}\right): i=1, \ldots, n\right\}$, where $y_{i}$ is the univariate response, $\mathbf{x}_{i}$ is a $p_{1} \times 1$ vector of completely observed covariates, and $\mathbf{z}_{i}$ is a $p_{2} \times 1$ vector of partially observed covariates. We use $\mathbf{r}_{i}$, a $p_{2} \times 1$ random vector, to indicate the missingness of $\mathbf{z}_{i}: r_{i k}=1$ if $z_{i k}$ is observed, and $r_{i k}=0$ if $z_{i k}$ is missing, where $r_{i k}$ and $z_{i k}$ are the $k$ th component of $\mathbf{r}_{i}$ and $\mathbf{z}_{i}$, respectively.

We use $p\left(D_{c} \mid \boldsymbol{\eta}\right)$ to denote the complete-data density function with $\boldsymbol{\eta}$ being the vector of all unknown parameters. One way of modeling the complete-data density is to use three layers of conditional densities as follows:

$$
p\left(D_{c} \mid \eta\right)=\prod_{i=1}^{n} p\left(y_{i} \mid \mathbf{x}_{i}, \mathbf{z}_{i}, \beta, \tau\right) p\left(\mathbf{z}_{i} \mid \mathbf{x}_{i}, \alpha\right) p\left(\mathbf{r}_{i} \mid \mathbf{x}_{i}, \mathbf{z}_{i}, y_{i}, \xi\right),
$$

where $(\boldsymbol{\beta}, \tau)$ are the parameters for the conditional distribution of $y_{i}$ given $\left(\mathbf{x}_{i}, \mathbf{z}_{i}\right), \boldsymbol{\alpha}$ is the parameter vector for the covariate distribution $p\left(\mathbf{z}_{i} \mid \mathbf{x}_{i}, \boldsymbol{\alpha}\right)$, and $\boldsymbol{\xi}$ is the parameter vector for modeling the missing data mechanism $p\left(\mathbf{r}_{i} \mid \mathbf{x}_{i}, \mathbf{z}_{i}, y_{i}, \boldsymbol{\xi}\right)$. The three sets of parameters are assumed distinct from one another, and $\boldsymbol{\eta}=\left(\boldsymbol{\beta}^{\prime}, \tau, \boldsymbol{\alpha}^{\prime}, \boldsymbol{\xi}^{\prime}\right)^{\prime}$.

We need to specify each of the three components in equation (1). Under the GLM, $y_{i}$ given $\left(\mathbf{x}_{i}, \mathbf{z}_{i}\right)$ has a density in the exponential family

$$
p\left(y_{i} \mid \mathbf{x}_{i}, \mathbf{z}_{i}, \beta, \tau\right)=\exp \left\{a_{i}^{-1}(\tau)\left[y_{i} \theta_{i}(\beta)-b\left(\theta_{i}(\beta)\right)\right]+c\left(y_{i}, \tau\right)\right\},
$$

$i=1, \ldots, n$, indexed by the canonical parameter $\theta_{i}$ and the scale parameter $\tau$, where the functions $b(\cdot)$ and $c(\cdot, \cdot)$ determine a particular distribution in the class. The functions $a_{i}(\tau)$ are commonly of the form $a_{i}(\tau)=\tau^{-1} k_{i}^{-1}$, where the $k_{i}$ 's are known weights. Further, the $\theta_{i}$ 's satisfy the equations $\theta_{i}=\theta\left(\mu_{i}\right)$, and $\mu_{i}=g\left(\left(\mathbf{x}_{i}{ }^{\prime}, \mathbf{z}_{i}{ }^{\prime}\right) \boldsymbol{\beta}\right)$ are the components of $\boldsymbol{\mu}=E(\mathbf{y} \mid \mathbf{x}, \mathbf{z}, \boldsymbol{\beta}, \tau)$, where $g$ $(\cdot)$ is a known link function and $\boldsymbol{\beta}=\left(\beta_{0}, \beta_{1}, \ldots, \beta_{p}\right)^{\prime}$ is a $(p+1) \times 1$ vector of regression coefficients, in which $p=p_{1}+p_{2}$.

Next, we need to specify a distribution for $\mathbf{z}_{i}$ given $\mathbf{x}_{i}$. We suggest specifying the covariate distribution via a sequence of one-dimensional conditional distributions:

$$
\begin{aligned}
p\left(\mathbf{z}_{i} \mid \mathbf{x}_{i}, \alpha\right)= & p\left(z_{i p_{2}} \mid z_{i\left(p_{2}-1\right)}, \ldots, z_{i 1}, \mathbf{x}_{i}, \alpha\right) \\
& \times \cdots p\left(z_{i 2} \mid z_{i 1}, \mathbf{x}_{i}, \alpha\right) \times p\left(z_{i 1} \mid \mathbf{x}_{i}, \alpha\right) .
\end{aligned}
$$

We typically assume specific parametric forms for these one-dimensional conditional distributions. This strategy allows much flexibility in the specification of the joint covariate distribution and has the potential of reducing the number of nuisance parameters (Lipsitz and Ibrahim, 1996; Ibrahim, Lipsitz, and Chen, 1999). Furthermore, we model the missing data mechanism using a sequence of one-dimensional conditional distributions as 


$$
\begin{aligned}
p\left(\mathbf{r}_{i} \mid y_{i}, \mathbf{x}_{i}, \mathbf{z}_{i}, \xi\right)= & p\left(r_{i p_{2}} \mid r_{i\left(p_{2}-1\right)}, \ldots, r_{i 1}, \mathbf{x}_{i}, \mathbf{z}_{i}, y_{i}, \xi\right) \\
& \times \cdots \times p\left(r_{i 2} \mid r_{i 1}, \mathbf{x}_{i}, \mathbf{z}_{i}, y_{i}, \xi\right) \\
& \times p\left(r_{i 1} \mid \mathbf{x}_{i}, \mathbf{z}_{i}, y_{i}, \xi\right)
\end{aligned}
$$

Because $r_{i j}$ is binary, a sequence of logistic regressions is commonly used.

\section{Local Influence}

We will develop a local influence method for carrying out sensitivity analyses of various assumptions of a GLM with missing covariates. Specifically, we will address three important issues related to local influence methods: perturbation schemes for perturbing the distributions for each component in equation (1), the appropriate choice of a perturbation vector, and the development of influence measures.

\subsection{A Simple Example}

Throughout this section, we examine a linear regression model with one missing covariate to illustrate our methodological development. We consider the model

$$
y_{i}=\beta_{0}+\beta_{1} x_{i}+\beta_{2} z_{i}+\epsilon_{i},
$$

where $\epsilon_{\mathrm{i}} \sim N(0, \tau)$. We assume that $y_{i}$ and $x_{i}$ are completely observed for $i=1, \ldots, n$, but the covariate $z_{i}$ may be missing for some cases. We also assume that $\left(z_{i} \mid x_{i}, \boldsymbol{\alpha}\right) \sim N\left(\alpha_{0}+\alpha_{1} x_{i}, \alpha_{2}\right)$, where $\alpha=\left(\alpha_{0}, \alpha_{1}, \alpha_{2}\right)$. We let $r_{i}=1$ if $z_{i}$ is missing and $r_{i}=0$ if $z_{i}$ is observed. Furthermore, we assume that the $z_{i}$ 's are MAR with missing data mechanism

$$
p\left(r_{i}=1 \mid y_{i}, x_{i}, z_{i}\right)=\frac{\exp \left(\xi_{0}+\xi_{1} y_{i}+\xi_{2} x_{i}\right)}{1+\exp \left(\xi_{0}+\xi_{1} y_{i}+\xi_{2} x_{i}\right)} .
$$

We introduce various perturbations to perturb $p\left(D_{c} \mid \boldsymbol{\eta}\right)$ and then we assess the sensitivity of each perturbation scheme to the proposed model and associated statistical inference. As an illustration, we consider four perturbations as follows. These perturbations illustrate two different types of perturbation schemes, which we discuss in the next subsection. The first is to perturb the variances of $\epsilon_{i}$ such that

$$
\operatorname{Var}\left(\epsilon_{1}, \ldots, \epsilon_{n}\right)=\tau \operatorname{diag}\left(1 / \omega_{1}, \ldots, 1 / \omega_{n}\right) .
$$

Throughout, we let $\boldsymbol{\omega}^{0}$ denote no perturbation. In this case, $\boldsymbol{\omega}^{0}=\mathbf{1}_{n}$ is an $n \times 1$ vector with all 1 's. This perturbation is designed to assess the homogeneous variance assumption of the $\epsilon_{i}$ 's. The second is to introduce a perturbation to $z_{i}$ to assess the linear relationship between $y_{i}$ and $z_{i}$ such that

$$
y_{i}=\beta_{0}+\beta_{1} x_{i}+\beta_{2}\left(z_{i}+\omega_{i}\right)+\epsilon_{i},
$$

for $i=1, \ldots, n$. In this case, $\omega^{0}=\mathbf{0}_{n}$, which is an $n \times 1$ vector with all 0 's.

The third is to extend the MAR assumption such that 


$$
p\left(r_{i}=1 \mid y_{i}, x_{i}, z_{i}, \omega\right)=\frac{\exp \left(\xi_{0}+\xi_{1} y_{i}+\xi_{2} x_{i}+\omega z_{i}\right)}{1+\exp \left(\xi_{0}+\xi_{1} y_{i}+\xi_{2} x_{i}+\omega z_{i}\right)} .
$$

If $\omega \neq 0$, then the missing data mechanism is NMAR. This strategy for checking NMAR is similar to that of Verbeke et al. (2001) in the context of longitudinal data. Thus, equation (9) explores the influence of perturbing the MAR assumption $\left(\omega^{0}=0\right)$ in the direction of NMAR. We emphasize here that formal tests for MAR or NMAR missingness should be approached with great caution, although they might be possible. Our main goal here and throughout this article is to use local influence methods to carry out sensitivity analyses to assess the effect of perturbing the given GLM with MAR covariates in the direction of NMAR. An alternative to equation (9) is the individual-specific infinitesimal perturbation as used in Verbeke et al. (2001), Hens et al. (2005), and Jansen et al. (2006), which is given by

$$
p\left(r_{i}=1 \mid y_{i}, x_{i}, z_{i}, \omega\right)=\frac{\exp \left(\xi_{0}+\xi_{1} y_{i}+\xi_{2} x_{i}+\omega_{i} z_{i}\right)}{1+\exp \left(\xi_{0}+\xi_{1} y_{i}+\xi_{2} x_{i}+\omega_{i} z_{i}\right)}
$$

This can provide insight into which case may have large influence.

The fourth perturbation extends the linear relationship between $z_{i}$ and $x_{i}$ such that $\left(z_{i} \mid x_{i}, \boldsymbol{\alpha}\right) \sim$ $N\left(\alpha_{0}+\alpha_{1} x_{i}+g\left(x_{i}\right), \alpha 2\right)$ for $i=1, \ldots, n$, where $g(\cdot)$ is an unknown function. For instance, we may approximate $g(x)$ using a set of $m$ basis functions (e.g., Fourier series, B-splines) $B 1(x)$, $\ldots, B_{m}(x)$ such that $g(x) \approx \sum_{j=1}^{m} \omega_{j} B_{j}(x)$. Thus, we obtain

$$
\left(z_{i} \mid x_{i}, \alpha\right) \sim\left(\alpha_{0}+\alpha_{1} x_{i}+\sum_{j=1}^{m} \omega_{j} B_{j}\left(x_{i}\right), \alpha_{2}\right)
$$

for $i=1, \ldots, n$. In equation (11), we are interested in assessing whether there is a nonlinear relationship between the covariate $z_{i}$ and $x_{i}$. In this case, $\boldsymbol{\omega}^{0}=\mathbf{0}_{m}$.

\subsection{Perturbation Schemes}

We formally define two classes of perturbation schemes: the single-case and the global perturbation scheme. Let $\omega=\left(\omega_{1}, \ldots, \omega_{m}\right) \in R^{m}$ be a perturbation vector for the completedata density $p\left(D_{c} \mid \boldsymbol{\eta}\right)$. We use $p\left(D_{c} \mid \boldsymbol{\eta}, \boldsymbol{\omega}\right)$ to denote the perturbed complete-data density such that $\int p\left(D_{c} \mid \boldsymbol{\eta}, \boldsymbol{\omega}\right) d D_{c}=1$ and $p\left(D_{c} \mid \boldsymbol{\eta}, \boldsymbol{\omega}^{0}\right)=p\left(D_{c} \mid \boldsymbol{\eta}\right)$. To assess the local influence of a model perturbation, we are primarily interested in the behavior of $p\left(D_{c} \mid \boldsymbol{\eta}, \boldsymbol{\omega}\right)$ as a function of $\boldsymbol{\omega}$ around $\omega^{0}$. We set $\boldsymbol{\eta}$ at a given value (e.g., the maximum likelihood estimate).

The single-case perturbation scheme refers to any scheme that independently perturbs individual observations (Verbeke et al., 2001). The single-case perturbation is mainly for identifying influential observations. Specifically, the perturbed complete-data density is

$$
p\left(D_{c} \mid \eta, \omega\right)=\prod_{i=1}^{n} p\left(\mathbf{x}_{i}, \mathbf{z}_{i}, \mathbf{r}_{i}, y_{i} \mid \eta, \omega_{i}\right)
$$

where $\omega=\left(\omega_{1}, \ldots, \omega_{n}\right)$ and $\omega_{i}$ denotes the perturbation to the $i$ th observation. Such perturbation schemes, for example, include case weights for each of the three components of equation (1), perturbing individual components of $\left(\mathbf{x}_{i}, \mathbf{z}_{i}, \mathbf{r}_{i}\right)$ and perturbing individual components (or 
multiple components) of $\mathbf{r}_{i}$. Perturbations (7), (8), and (10) of the previous subsection belong to such a class.

The global perturbation scheme refers to any scheme that perturbs all observations simultaneously (Troxel et al., 2004; Copas and Eguchi, 2005). The global perturbation is mainly for assessing the robustness of model assumptions to small perturbations. Specifically, the perturbed complete-data density is

$$
p\left(D_{c} \mid \eta, \omega\right)=\prod_{i=1}^{n} p\left(\mathbf{x}_{i}, \mathbf{z}_{i}, \mathbf{r}_{i}, y_{i} \mid \eta, \omega\right),
$$

where $\omega=\left(\omega_{1}, \ldots, \omega_{m}\right)$ is shared by all the observations. Such a perturbation scheme includes the perturbation of each of the three components of equation (1) and simultaneous perturbations of the three components of equation (1), among many others. The number of components in $\omega$ can be as small as one, such as perturbation (9) and other examples (Gustafson, 2001; Troxel et al., 2004; Copas and Eguchi, 2005; Zhu et al., 2007). Perturbation (11) is also a global perturbation scheme, in which $m$ in the perturbation can increase with $n$.

\subsection{Appropriate Perturbation}

We develop a new geometric framework to address the issue of selecting an appropriate perturbation scheme for equation (1). This issue is central to the development of the local influence approach, because arbitrarily perturbing a model may lead to inappropriate inference about the cause (e.g., influential observations) of a large effect.

The perturbed model $p\left(D_{c} \mid \boldsymbol{\eta}, \boldsymbol{\omega}\right)$ has a natural geometrical structure. The perturbed model $M=\left\{p\left(D_{c} \mid \boldsymbol{\eta}, \boldsymbol{\omega}\right): \boldsymbol{\omega} \in R^{m}\right\}$ can be regarded as an $m$-dimensional manifold. At each $\boldsymbol{\omega} \in$ $M$, there is a tangent space $T_{\omega}$ of $M$ spanned by $m$ functions $\partial_{\omega_{j}} l_{c}(\omega)$, where $l_{c}(\omega)=\log p$ $\left(D_{c} \mid \boldsymbol{\eta}, \boldsymbol{\omega}\right)$. The $m^{2}$ quantities $g_{j k}(\boldsymbol{\omega})=\mathrm{E}_{\boldsymbol{\omega}}\left[\partial_{\omega_{j}} l_{c}(\boldsymbol{\omega}) \partial_{\omega_{k}} l_{c}(\boldsymbol{\omega})\right], \mid j, k=1, \ldots, m$ form the metric tensor of $M$, in which $\mathrm{E}_{\omega}$ denotes the expectation taken with respect to $p\left(D_{c} \mid \boldsymbol{\eta}, \boldsymbol{\omega}\right)$, and the metric matrix $G(\omega)=\left(g_{i j}(\omega)\right)$ is the Fisher information matrix with respect to the perturbation vector $\omega$ (Figure 1).

An appropriate perturbation to equation (1) requires that $G\left(\omega^{0}\right)=\operatorname{diag}\left(g_{11}\left(\omega^{0}\right), \ldots, g_{m} m\right.$ $\left.\left(\omega^{0}\right)\right)$. The elements of $G(\omega)$ measure the amount of perturbation introduced by all components of the perturbation vector $\omega$. The $g_{i i}(\omega)$ can be interpreted as the amount of perturbation introduced by $\omega_{i}$, whereas $r_{i j}(\omega)=g_{i j}(\omega) / \sqrt{g_{i i}(\omega) g_{j j}(\omega)}$ indicates an association between $\omega_{i}$ and $\omega_{j}$. For a diagonal matrix $G(\omega)$, all components of $\omega$ may be regarded as being orthogonal to each other in the perturbed model (Cox and Reid, 1987), and therefore it becomes easy to pinpoint the cause of a large effect. In applications, although $G\left(\omega^{0}\right)$ may not be diagonal, we can always choose a new perturbation vector $\tilde{\omega}$, defined by

$$
\tilde{\omega}(\omega)=\omega^{0}+c^{-1 / 2} G\left(\omega^{0}\right)^{1 / 2}\left(\omega-\omega^{0}\right)
$$

such that $G(\tilde{\omega})$ evaluated at $\omega^{0}$ equals $c \mathbf{I}_{m}$, where $c>0$.

For the single-case perturbation scheme (12), we have $g_{j k}(\boldsymbol{\omega})=\delta_{j k} \mathrm{E}_{\boldsymbol{\omega}}\left[\partial_{\omega_{j}} l_{c, j}(\boldsymbol{\omega})\right]^{2}$, for $j, k=$ $1, \ldots, n$, where $\delta_{j k}$ is the Kronecker delta and $l_{c, j}(\boldsymbol{\omega})=\log p\left(\mathbf{d}_{j} \mid \boldsymbol{\eta}, \omega_{j}\right)$. The diagonal structure of $G(\omega)=\left(g_{j k}(\omega)\right)$ indicates that all components of $\omega$ are orthogonal to each other. Furthermore, if $p\left(\mathbf{d}_{i} \mid \boldsymbol{\eta}, \omega_{i}\right)$ is invariant across all $i$, then $G(\boldsymbol{\omega})=g_{11}(\boldsymbol{\omega}) \mathbf{I}_{n}$, which indicates that different components of $\omega$ have the same influence on the corresponding distributions. 
For the global perturbation scheme, we have $g_{j k}(\omega)=-\sum_{i=1}^{n} E_{\omega}\left[\partial_{\omega_{j} \omega_{k}}^{2} \ell_{c, i}(\omega)\right]$. Although $\omega$ may not be appropriate, we can choose a new perturbation $\tilde{\omega}=\boldsymbol{\omega}^{0}+G\left(\boldsymbol{\omega}^{0}\right)^{1 / 2}\left(\boldsymbol{\omega}-\boldsymbol{\omega}^{0}\right)$ such that $G$ $\left(\tilde{\omega}^{0}\right)=\mathbf{I}_{n}$. Thus, $\tilde{\boldsymbol{\omega}}$ is an appropriate perturbation at least at $\tilde{\boldsymbol{\omega}}^{0}=\boldsymbol{\omega}^{0}$. For instance, we consider the perturbation (11) to the model in Section 3.1. It can be shown that

$$
\begin{aligned}
-\partial_{\omega_{j} \omega_{k}}^{2} \ell_{c}(\omega) & =\alpha_{2}^{-1} B_{j}\left(x_{i}\right) B_{k}\left(x_{i}\right) \quad \text { and } \quad g_{j k}(\omega) \\
& =\alpha_{2}^{-1} \sum_{i=1}^{n} \int B_{j}\left(x_{i}\right) B_{k}\left(x_{i}\right) p\left(x_{i} \mid \alpha\right) d x_{i},
\end{aligned}
$$

where $p\left(x_{i} \mid \boldsymbol{\alpha}\right)$ is the distribution of $x_{i}$. If $\left\{B_{j}(x): j=1, \ldots, m\right\}$ forms an orthonormal basis with respect to $p(x \mid \boldsymbol{\alpha})$, then $G(\boldsymbol{\omega})$ is just an $m \times m$ identity matrix. However, because the $x_{i}$ 's are always observed, we can always treat $x_{i}$ as fixed and approximate $g_{j k}(\boldsymbol{\omega})$ using

$g_{j k}(\omega)=\alpha_{2}^{-1} \Sigma_{i=1}^{n} B_{j}\left(x_{i}\right) B_{k}\left(x_{i}\right)$.

\subsection{Influence Measures}

3.4.1 First-order Influence Measures-We consider a $b \times 1$ objective function $f(\omega): M$ $\rightarrow R^{b}$ such as the maximum likelihood estimate of $\boldsymbol{\eta}$ (Copas and Eguchi, 2001, 2005; Gustafson, 2001; Troxel et al., 2004). The objective function $f(\omega)$ defines the aspect of inference of interest for sensitivity analysis. Let $\boldsymbol{\omega}(t)$ be a geodesic on $M$ with $\boldsymbol{\omega}(0)=\boldsymbol{\omega}^{0}$ and $\left.\partial_{t} \boldsymbol{\omega}(t)\right|_{t=0}=\mathbf{h} \in$ $R^{m}$. It follows from a Taylor's series expansion that $f(\boldsymbol{\omega}(t))=f(\boldsymbol{\omega}(0))+\dot{f}_{h}(0) t+O\left(t^{2}\right)$, where

$f_{\mathbf{h}}(0)=\Sigma_{j} \partial_{\omega_{j}} f\left(\omega^{0}\right) h_{j}=\nabla_{f}^{\prime} \mathbf{h}$. If $\nabla f \neq 0$, then the first-order term $\dot{f}_{\mathrm{h}}(0)$ mainly characterizes the local influence of a perturbation vector $\omega$ to a model.

We introduce a first-order influence measure to assess the local influence of minor perturbations when $\nabla_{f}=0$. The first-order influence measure (FI) in the direction $\mathbf{h} \in R^{m}$ is $\mathrm{FI}_{f, \mathbf{h}}=\mathrm{FI}_{f\left(\omega^{0}\right), \mathbf{h}}=\frac{\mathbf{h}^{\prime} \nabla_{f} W_{f} \nabla_{f}^{\prime} \mathbf{h}}{\mathbf{h}^{\prime} G \mathbf{h}}$, where $G=G\left(\boldsymbol{\omega}^{0}\right)$ and $W_{f}$ is a positive semi-definite matrix.

Although $\omega$ may not be an appropriate perturbation, we can always use the appropriate perturbation $\boldsymbol{\omega}(\boldsymbol{\omega})$ in equation (14), which yields

$$
\mathrm{FI}_{f(\tilde{\omega}) \mathbf{h} \mathbf{h} \tilde{\omega}=\omega^{0}}=\frac{\mathbf{h}^{\prime} G^{-1 / 2} \nabla_{f} W_{f} \nabla_{f}^{\prime} G^{-1 / 2} \mathbf{h}}{\mathbf{h}^{\prime} \mathbf{h}} .
$$

The maximum value of $\mathrm{FI}_{f, h}$ equals the principal eigenvalue of $G^{-1 / 2} \nabla_{f} W_{f} \nabla_{f}^{\prime} G^{-1 / 2}$, which quantifies the largest degree of local influence of $\omega$ to a statistical model, while the corresponding eigenvector of $G^{-1 / 2} \nabla_{f} W_{f} \nabla_{f}^{\prime} G^{-1 / 2}$, denoted by $\mathbf{h}_{\text {max }}$, can be used either for identifying influential observations for single-case perturbations or for identifying influential directions for global perturbations (Copas and Eguchi, 2005). The $\mathbf{h}_{\max }$ is the largest perturbation direction for $f(\boldsymbol{\omega})$.

3.4.2 Maximum Likelihood Estimate as the Objective Function-Let $D_{o}$ denote the observed data. We consider $\hat{\boldsymbol{v}}_{0}(\boldsymbol{\omega})=\left(\hat{\beta}_{o}(\boldsymbol{\omega}), \hat{\boldsymbol{\alpha}_{o}}(\boldsymbol{\omega}), \hat{\boldsymbol{\xi}}_{o}(\boldsymbol{\omega})\right)^{\prime}$, which is the maximum likelihood estimate of $v$ based on the perturbed observed-data density. The perturbed observed-data density, denoted by $p\left(D_{o} \mid \boldsymbol{v}, \boldsymbol{\omega}\right)$, is associated with the perturbed complete-data density through $p\left(D_{o} \mid \boldsymbol{v}, \boldsymbol{\omega}\right)=\int p\left(D_{c} \mid \boldsymbol{v}, \boldsymbol{\omega}\right) d D_{m}$. It can be shown that 


$$
\partial_{\omega} \widehat{\eta}_{o}\left(\omega^{0}\right)=\left.I_{\eta, o}^{-1} \Delta_{o}(\eta, \omega)\right|_{\eta=\widehat{\eta}, \omega=\omega^{0}},
$$

where $I_{\eta, o}=-\partial_{\eta}^{2} \quad \log \quad p\left(D_{o} \mid \eta\right)$ and $\Delta_{o}(\eta, \omega)=\partial_{\eta \omega}^{2} \quad \log \quad p\left(D_{o} \mid \eta, \omega\right)$. Then, the asymptotic bias in the estimate of $\boldsymbol{v}$ is $\partial_{\omega} \hat{v}_{o}\left(\omega^{0}\right)\left(\omega-\omega^{0}\right)$ under $p\left(D_{o} \mid \boldsymbol{v}, \omega\right)$.

We choose $\boldsymbol{\eta}^{\wedge}{ }_{o}$ as the object of interest and set $W_{f}=I_{\boldsymbol{\eta}^{\wedge}, o}$. We can show that

$$
\mathrm{FI}_{\widehat{\eta}_{o}(\tilde{\omega}), \mathbf{h}}=\mathbf{h}^{\prime} G^{-1 / 2} \Delta_{o}\left(\widehat{\eta}, \omega^{0}\right)^{\prime} I_{\bar{\eta}, o}^{-1} \Delta_{o}\left(\widehat{\eta}, \omega^{0}\right) G^{-1 / 2} \mathbf{h}
$$

where $\mathbf{h}^{\prime} \mathbf{h}=1$. If $G=\mathbf{I}_{m}$, then it can be shown that $\mathrm{FI}_{\boldsymbol{\eta}^{\wedge}{ }_{\mathbf{o}}\left(\boldsymbol{\omega}^{\sim}\right), \mathbf{h}}$ is the same as Cook's (1986) local influence measure based on the likelihood displacement. Finally, for most GLMs with

missing covariate data, computing the matrix $G^{-1 / 2} \Delta_{o}\left(\widehat{\eta}, \omega^{0}\right)^{\prime} I_{\widehat{\eta}, o}^{-1} \Delta_{o}\left(\widehat{\eta}, \omega^{0}\right) G^{-1 / 2}$ involves the computation of $G, \Delta_{o}\left(\eta^{\wedge}, \omega^{0}\right)$, and $I_{\eta, o}$, which can be expressed as expectations with respect to the conditional distribution of $\mathbf{Z}_{m, i}$ given $\mathbf{d}_{o, i}$ and hence be computed using Markov chain Monte Carlo methods. For the single-case perturbation in equation (12), we obtain $G\left(\omega^{0}\right)=$ $g_{11}\left(\omega^{0}\right) \mathbf{I}_{n}$ and the $i$ th column of $\Delta_{o}\left(\boldsymbol{\eta}, \boldsymbol{\omega}^{0}\right)$, denoted by $\delta_{\eta, i}$, is given by $\partial_{\eta \omega_{i}}^{2}\left\{\log \int p\left(\mathbf{d}_{i} \mid \eta, \omega_{i}\right) d \mathbf{z}_{m, i}\right\}$. Thus,

$$
\mathrm{FI}_{\widehat{\eta}_{o}(\tilde{\omega}), \mathbf{h}}=g_{11}\left(\omega^{0}\right)^{-1} \mathbf{h}^{\prime} \Delta_{o}\left(\widehat{\eta}, \omega^{0}\right)^{\prime} I_{\widehat{\eta}, o}^{-1} \Delta_{o}\left(\widehat{\eta}, \omega^{0}\right) \mathbf{h}
$$

In particular, for the $i$ th observation, $\mathrm{FI}_{\widehat{\eta}_{o}}(\tilde{\omega}), \mathbf{e}_{i}=g_{11}\left(\omega^{0}\right)^{-1} \delta_{\widehat{\eta}_{i},}^{\prime} I_{\widehat{\eta}^{\prime}, o}^{-1} \delta_{\tilde{\eta}, i}$, and $\sum_{i=1}^{n} \mathrm{FI}_{\widehat{\eta}_{o}}(\tilde{\omega}), \mathbf{e}_{i}=g_{11}\left(\omega^{0}\right)^{-1} \operatorname{tr}\left\{\sum_{i=1}^{n} \delta_{\widehat{\eta}, i} \delta_{\widehat{\eta}, i}^{\prime} I_{\widehat{\eta}, o}^{-1}\right\}$. Under some mild conditions, $\Sigma_{i=1}^{n} \delta_{\widehat{\eta}, i} \delta_{\widehat{\eta}, i}^{\prime} / n$ and $I_{\boldsymbol{\eta}^{\wedge}, o} / n$ converge in probability to $J_{o}$ and $I_{o}$, respectively. Therefore, ${ }_{i=1}^{n} \mathrm{FI}_{\bar{\eta}_{0}}(\tilde{\omega}), \mathbf{e}_{i}$ is a direct estimate of $\lambda_{0}=\operatorname{tr}\left(J_{o} I_{o}^{-1}\right) g_{11}\left(\omega^{0}\right)^{-1}$. Under exchangeability of the observations, each $\mathrm{FI}_{\boldsymbol{\eta}^{\wedge}{ }_{o}\left(\omega^{\sim}\right), \mathbf{e}_{i}}$ should be around its mean $\lambda_{0}$. However, in real applications, if a particular $\mathrm{FI}_{\boldsymbol{\eta}^{\wedge} o}\left(\omega^{\sim}\right), \mathbf{e}_{i}$ is much larger than $\lambda_{0}$, then this observation may be regarded as an influential case.

3.4.3 Likelihood Ratio as the Objective Function-We consider $f_{l r}(\omega)=\log p\left(D_{\mathrm{o}} \mid\right.$ $\left.\boldsymbol{\eta}^{\wedge}, \boldsymbol{\omega}\right)-\log p\left(D_{\mathrm{o}} \mid \boldsymbol{\eta}^{\wedge}\right)$ as our objective function. For the single-case perturbation in equation (12), we can obtain that $G\left(\omega^{0}\right)=g_{11}\left(\omega^{0}\right) \mathbf{I}_{n}$ and

$$
\begin{aligned}
\partial_{\omega_{i}} f_{l r}(\omega) & =\partial_{\omega_{i}} \log p\left(\mathbf{d}_{o, i} \widehat{\eta}, \omega_{i}\right) \\
& =E\left[\partial_{\omega_{i}} \log p\left(\mathbf{d}_{i} \mid \widehat{\eta}, \omega_{i}\right) \mid \mathbf{d}_{o, i}, \widehat{\eta}\right]
\end{aligned}
$$

for $i=1, \ldots, n$, where the expectation is taken with respect to the conditional distribution of $\mathbf{z}_{m, i}$ given $\mathbf{d}_{o, i}$. Thus, by setting $W_{f l r}=1$, we get $\mathrm{FI}_{f l r}(\boldsymbol{\omega}), \mathbf{h}=g_{11}\left(\boldsymbol{\omega}^{0}\right)^{-} 1 \mathbf{h}^{\prime} \nabla_{f l r} \nabla_{f l r}^{\prime} \mathbf{h}$. For the $i$ th observation, we have $\mathrm{FI}_{f l r}(\boldsymbol{\omega}), \mathbf{e}_{i}=g_{11}\left(\boldsymbol{\omega}^{0}\right)^{-} 1\left\{\partial_{\omega i} f l r\left(\omega^{0}\right)\right\}^{2}$. If a particular $\mathrm{FI}_{f l r}(\boldsymbol{\omega}), \mathbf{e}_{i}$ is much larger than the mean of all $\mathrm{FI}_{f l r}(\omega), \mathbf{e}_{\mathbf{i}}$ 's, then the $i$ the observation can be regarded as influential. 
For the global-case perturbation in equation (13), we define

$\log p\left(D_{o} \sqrt{\eta}, \omega\right)=\Sigma_{i=1}^{n} \log p\left(\mathbf{d}_{o, i}, \widehat{\eta}, \omega\right)$. Direct calculation leads to

$$
\begin{aligned}
\nabla_{f_{l r}} & =\sum_{i=1}^{n} \partial_{\omega} \log p\left(\mathbf{d}_{o, i}, \widehat{\eta}, \omega^{0}\right) \\
& =\sum_{i=1}^{n} E\left[\partial_{\omega} \log p\left(\mathbf{d}_{i}, \widehat{\eta}, \omega^{0}\right) \mid \mathbf{d}_{o, i}, \widehat{\eta}\right]
\end{aligned}
$$

Setting $W_{f l r}=1$ and choosing $\omega^{\sim}$ in equation (14), we have $\mathrm{FI}_{f_{l r}(\tilde{\omega}), \mathbf{h}}=\mathbf{h}^{\prime} G^{-1 / 2} \nabla_{f_{l r}} \nabla_{f_{l r}}^{\prime} G^{-1 / 2} \mathbf{h}$,

where $\mathbf{h}^{\prime} \mathbf{h}=1$. The maximum value is the principal eigenvalue $\mathrm{FI}_{f_{l r}(\tilde{\omega}), \mathbf{h}_{\max }}=\nabla_{f_{l r}^{\prime}}^{\prime} G^{-1} \nabla_{f_{l r}}$ and its corresponding $\mathbf{h}_{\max }$ is $G^{-1 / 2} \nabla_{f l r} /\left\|G^{-1 / 2} \nabla_{f l r}\right\|$. Moreover, under some mild conditions $\nabla_{f_{l r}}^{\prime} G^{-1} \nabla_{f_{l r}}$ can be used as a test statistic for testing $H_{0}: \omega=0$. Under $H_{0}: \boldsymbol{\omega}=0$, it can be shown that $\nabla_{f_{l r}} / \sqrt{n}$ converges in distribution to a Gaussian distribution with zero mean and covariance matrix $\Sigma_{f l r}$ as $n \rightarrow \infty$. Thus, $\nabla_{f_{l r}}^{\prime} \Sigma_{f_{l r}}^{-1 / 2} \Sigma_{f_{l r}}^{1 / 2} G^{-1} \Sigma_{f_{l r}}^{1 / 2} \Sigma_{f_{l r}}^{-1 / 2} \nabla_{f_{l r}}$ converges in distribution to a weighted chi-squared distribution as $n \rightarrow \infty$. Therefore, we may use the asymptotic distribution of $\nabla_{f_{l r}}^{\prime} G^{-1} \nabla_{f_{l r}}$ to characterize the asymptotic behavior of the influence measures $\mathrm{FI}_{f l r}\left(\omega^{\sim}\right), \mathbf{h} \cdot$

\section{Simulation Studies}

We applied the proposed local influence measures to several simulated datasets in which various assumptions were misspecified to examine their performance. First, we applied two single-case perturbation schemes to simulated datasets in each of which an outlier was added. We expected that both schemes could detect the outlier both in the response and in the covariates. Secondly, we used several perturbation schemes to examine the functional form of the missing data mechanism and to assess the relationship between the response and covariates.

We generated 500 simulated datasets from model (5) with $n=100, \beta_{0}=\beta_{1}=\beta_{2}=1$ and $\tau=1$. Moreover, $\left(x_{i}, z_{i}\right)$ were generated from a $N_{2}\left(\mathbf{0}, \mathbf{I}_{2}\right)$ distribution. We also assumed an MAR missing data mechanism for $z_{i}$ given by

$$
p\left(r_{i}=1 \mid x_{i}, z_{i}, y_{i}\right)=\frac{\exp \left(\xi_{0}+\xi_{1} x_{i}\right)}{1+\exp \left(\xi_{0}+\xi_{1} x_{i}\right)},
$$

with $\xi_{0}=-0.5$ and $\xi_{1}=1.0$, resulting in an average missingness fraction of $40 \%$. Then, we fit $y_{\mathrm{i}}=\beta_{0}+\beta_{1} x_{i}+\beta_{2} z_{i}+\epsilon_{i}$ with MAR $z_{i}$, and changed $y_{100}$ to $y_{100}+\delta$ with $\delta=1.0,2.0,3.0,4.0$, and 5.0 to add an outlier. We applied two single-case perturbation schemes. The first was to perturb the variance of $\epsilon_{i}$ such that $\operatorname{Var}\left(\epsilon_{1}, \ldots, \epsilon_{n}\right)=\tau \operatorname{diag}\left(1 / \omega_{1}, \ldots, 1 / \omega_{n}\right)$, where $\boldsymbol{\omega}^{0}=\mathbf{1}_{n}$ is an $n \times 1$ vector with all 1's. The second perturbation was to perturb the missing covariate $z_{i}$ such that $y_{i}=\beta_{0}+\beta_{1} x_{i}+\beta_{2}\left(z_{i}+\omega_{i}\right)+\epsilon_{i}$ for $i=1, \ldots, n$, where $\omega^{0}=\mathbf{0}_{n}$ is an $n \times 1$ vector with all 0 's. We calculated $\mathrm{FI}_{\boldsymbol{\eta}^{\wedge}}{ }_{o}\left(\omega^{0}\right), \mathbf{e}_{i}$ and $\mathrm{FI}_{f l r}\left(\omega^{0}\right), \mathbf{e}_{i}$ for both perturbations, and their values for the last case were larger than those for the rest of the cases, especially when $\delta$ is large. The first half of Table 2 summarizes the percentages of detecting the outlier using either non-robust methods with the sample mean and standard deviation ( $>$ mean $+2 \times \mathrm{SD}$ or $>$ mean $+3 \times \mathrm{SD})$ or robust methods with the sample median and median absolute deviation ( $>$ median $+2 \times$ MAD or $>$ median $+3 \times$ MAD) for different values of $\delta$. As expected, the percentage of detecting the outlier increases with $\delta$, and the results based on the robust methods are better compared to the non-robust methods. The threshold based on three standard deviations (SD or 
MAD) is not very different from using a threshold based on two standard deviations. Based on a simulated dataset, in which $\delta=4$, the index plots of the two influence measures (Figure 2) can effectively detect the outlier. Instead of having an outlier in the response, we examined a scenario with the presence of the outlier in the covariates. We changed $z_{100}$ to $z_{100}+\delta$ with $\delta=1.0,2.0,3.0,4.0$, and 5.0, and applied the same two single-case perturbation schemes. The values of $\mathrm{FI}_{\boldsymbol{\eta}^{\wedge} o}\left(\omega^{0}\right), \mathbf{e}_{i}$ and $\mathrm{FI}_{f l r}\left(\omega^{0}\right), \mathbf{e}_{i}$ for both perturbations for the last case were again larger than those for the rest of the cases, especially when $\delta$ is large. The second half of Table 2 lists the percentages of detecting the outlier using either non-robust or robust methods mentioned previously, which shows similar findings as when the outlier is in the response. Thus our local influence method can effectively detect the outlier in the covariates when $\delta$ is reasonably large.

Next, we explored the potential deviations of the MAR missing data mechanism in the direction of NMAR. We generated data from model (5) with $n=200, \beta_{0}=\beta_{1}=\beta_{2}=1, \tau=1,\left(x_{i}, z_{i}\right)$ were generated from a $N_{2}\left(\mathbf{0}, \mathbf{I}_{2}\right)$ distribution, and the following missing data mechanism for $z_{i}$ was assumed,

$$
p\left(r_{i}=1 \mid x_{i}, z_{i}, y_{i}\right)=\frac{\exp \left(\xi_{0}+\xi_{1} y_{i}+\xi_{2} x_{i}+a z_{i}\right)}{1+\exp \left(\xi_{0}+\xi_{1} y_{i}+\xi_{2} x_{i}+a z_{i}\right)},
$$

with $\xi_{0}=-1.8, \xi_{1}=1.0$, and $\xi_{2}=1.0$ being chosen to make the missing data fraction approximately $40 \%$ for various values of $a$. If $a \neq 0$, then the missing data mechanism is nonignorable. We fit $y_{i}=\beta_{0}+\beta_{1} x_{i}+\beta_{2} z_{i}+\epsilon_{i}$ with the MAR missing data mechanism given by

$$
p\left(r_{i}=1 \mid x_{i}, z_{i}, y_{i}\right)=\frac{\exp \left(\xi_{0}+\xi_{1} y_{i}+\xi_{2} x_{i}\right)}{1+\exp \left(\xi_{0}+\xi_{1} y_{i}+\xi_{2} x_{i}\right)} .
$$

Then, we applied a global perturbation given by

$$
p\left(r_{i}=1 \mid y_{i}, x_{i}, z_{i}, \omega\right)=\frac{\exp \left(\xi_{0}+\xi_{1} y_{i}+\xi_{2} x_{i}+\omega z_{i}\right)}{1+\exp \left(\xi_{0}+\xi_{1} y_{i}+\xi_{2} x_{i}+\omega z_{i}\right)} .
$$

The $\mathrm{FI}_{f l r}\left(\omega^{0}\right)$ were $0.084,1.448$, and 4.795 for $a=0,0.5$, and 1.0, respectively. From these results, we see that as $a$ increases, the influence measure of $\mathrm{FI}_{f l r}\left(\omega^{0}\right)$ also increases, which may suggest that an NMAR model is tenable for large $a$. We also used the corresponding singlecase perturbation given by

$$
p\left(r_{i}=1 \mid y_{i}, x_{i}, z_{i}, \omega\right)=\frac{\exp \left(\xi_{0}+\xi_{1} y_{i}+\xi_{2} x_{i}+\omega_{i} z_{i}\right)}{1+\exp \left(\xi_{0}+\xi_{1} y_{i}+\xi_{2} x_{i}+\omega_{i} z_{i}\right)}
$$

No large $\mathrm{FI}_{f l r}\left(\omega^{0}\right), e_{i}$ was observed for any $i$ even when $a$ is large. This result might suggest that this type of NMAR mechanism is not detectable using only $\mathrm{FI}_{f l r}\left(\omega^{0}\right), e_{i}$, the diagonal entries of $G^{-1 / 2} \nabla_{f_{l i}} W_{f_{l r}} \nabla_{f_{l r}}^{\prime} G^{-1 / 2}$, confirming the analyses in Jansen et al. (2006). However, we observed increases in the off-diagonal entries of $G^{-1 / 2} \nabla_{f_{l r}} W_{f_{l l}} \nabla_{f_{l r}}^{\prime} G^{-1 / 2}$ as $a$ increases, indicating influence through combinations of cases.

As noted in Hens et al. (2005) and Jansen et al. (2006), a local influence tool for the missing data mechanism is able to pick up anomalous features of cases that are not necessarily related to the missing data mechanism. To study this notion, we generated an original dataset from 
model (5) with $n=200, \beta_{0}=\beta_{1}=\beta_{2}=1, \tau=1$, where $\left(x_{i}, z_{i}\right)$ were generated from a $N_{2}\left(\mathbf{0}, \mathbf{I}_{2}\right)$ distribution, and MAR was assumed. Then we generated a perturbed dataset in which we added 20 to the responses of the last five cases. We fit $y_{\mathrm{i}}=\beta_{0}+\beta_{1} x_{i}+\beta_{2} z_{i}+\epsilon_{i}$ with the MAR missing data mechanism given by equation (22). The perturbation (24) identified the last five cases as influential. Thus single-case perturbation for the missing data mechanism is able to pick up some deviations in the data even though the deviations are different from the functional form of the missing data mechanism. The global perturbation (23) resulted in $\mathrm{FI}_{f l r}\left(\omega^{0}\right)=1.61$, a big qualitative change compared to $\mathrm{FI}_{f l r}\left(\omega^{0}\right)=0.011$ for the original dataset. These results may thus raise some concerns about the MAR assumption, and/or about the model as a whole.

We also examined whether our influence measures can assess the relationship between the response and the covariates of interest. We generated data from $y_{i}=1+x_{i}+z_{i}+c * z_{i}^{2}+\epsilon_{i}$ for $i=$ $1, \ldots, 100$, where $\epsilon_{i} \sim N(0,1)$ and $\left(x_{i}, z_{i}\right)$ were independently generated from a $N_{2}\left(\mathbf{0}, \mathbf{I}_{2}\right)$ distribution. The missing data mechanism was assumed MAR as in equation (20) with a $40 \%$ missingness fraction. We fit $y_{i}=\beta_{0}+\beta_{1} x_{i}+\beta_{2} z_{i}+\epsilon_{i}$ assuming MAR $z_{i}^{\prime}$ s, and thus the fitted model would be misspecified if $c \neq 0$. We considered a global perturbation scheme

$y_{i}=\beta_{0}+\beta_{1} x_{i}+\beta_{2} z_{i}+\Sigma_{j=1}^{m+3} \omega_{j} B_{j}\left(z_{i}\right)+\epsilon_{i}$, where the $B_{j}(z)$ are truncated polynomials of order 2 to 4 , given by $z^{2}, z^{3}, z^{4},\left(z-k_{1}\right)_{+}^{4}, \ldots,\left(z-k_{m}\right)_{+}^{4}$, where $k_{1}, \ldots, k_{m}$ are the $m=3$ prefixed knots. The principal eigenvalue of $\mathrm{FI}_{f l r}\left(\omega^{0}\right)$ was $0.582,13.675,24.535$, and 33.233 for $c=0,0.4,0.8$, and 1.2 , respectively. The principal eigenvalue of $\mathrm{FI}_{f l r}\left(\omega^{0}\right)$ was statistically significant at the $5 \%$ significance level ( $p$-value $=0.002$ ) for $c=0.8$, but not significant for $c<0.8$. Thus, the local influence measures are useful for detecting model misspecification in this example.

\section{Real Data Analysis}

\subsection{Quality-of-Life Data}

As mentioned in Section 1, the response variable for these data is the logarithm of the survival time, in which all cases are light censored. The dataset has 404 observations and the covariates are: physical ability $\left(z_{1}\right)$; mood $\left(z_{2}\right)$; indicator for treatment $\mathrm{A}$ (yes, no) $\left(x_{1}\right)$; indicator for treatment B (yes, no) $\left(x_{2}\right)$; indicator for treatment C (yes, no) $\left(x_{3}\right)$; age $\left(x_{4}\right)$; and language (English, otherwise) $\left(x_{5}\right)$. Among these seven covariates, $z_{1}$ has $13 \%$ missingness and $z_{2}$ has $31 \%$ missingness, and the remaining covariates are fully observed.

We fit a regression model $y_{i}=\mathbf{v}_{i}^{\prime} \beta+\epsilon_{i}$, where $\epsilon_{i} \sim N(0, \tau), \mathbf{v}_{i}^{\prime}=\left(1, z_{i 1}, z_{i 2}, x_{i 1}, \ldots, x_{i 5}\right)$ is the $1 \times$ 8 vector of covariates and $\boldsymbol{\beta}=\left(\beta_{0}, \beta_{1}, \ldots, \beta_{7}\right)^{T}$ are the corresponding regression coefficients. Because only the continuous covariates $z_{1}$ and $z_{2}$ have missing values, we assumed $\left(z_{i 1}, z_{i 2}\right) \sim$ $N_{2}\left(\boldsymbol{\alpha}_{1}, \boldsymbol{\alpha}_{2}\right)$, for $i=1, \ldots, n$. We assumed that the missing covariates are MAR and calculated the maximum likelihood estimates of $\left(\boldsymbol{\beta}, \tau, \boldsymbol{\alpha}_{1}, \boldsymbol{\alpha}_{2}\right)$ using the expectation-maximization (EM) algorithm.

To detect the influential cases, we employed two single-case perturbation schemes. The first was to perturb the variance of $\epsilon_{i}$ such that $\operatorname{Var}\left(\epsilon_{1}, \ldots, \epsilon_{n}\right)=\tau \operatorname{diag}\left(1 / \omega_{1}, \ldots, 1 / \omega_{n}\right)$. We calculated $\mathrm{FI}_{\hat{\eta} o}\left(\omega^{0}\right), \mathbf{e}_{i}$ and $\mathrm{FI}_{f l r}\left(\omega^{0}\right), \mathbf{e}_{i}$, and both indicated that cases 132 and 404 were very influential (Figures $3 \mathrm{a}$ and $\mathrm{b}$ ). The second perturbation was to simultaneously perturb the missing covariates $z_{i 1}$ and $z_{i 2}$ such that $y_{i}=\beta_{0}+\beta_{1}\left(z_{i 1}+\omega_{i}\right)+\beta_{2}\left(z_{i 2}+\omega_{i}\right)+\beta_{3} x_{i 1}+\cdots+$ $\beta_{7} x_{i 5}+\epsilon_{i}$. Again, cases 132 and 404 were very influential (Figures $3 \mathrm{c}$ and d). The response values of cases 132 and 404 are very small, compared to the rest of the cases.

Next, we were interested in sensitivity analyses regarding the MAR assumption in the direction of NMAR. First, we fit the model with a MAR missing data mechanism 
where $p\left(r_{i 1} \mid \mathbf{x}_{i}, y_{i}, \xi_{1}\right)=\frac{\exp \left(r_{i 1} f_{i 1}\right)}{1+\exp \left(f_{i 1}\right)}, p\left(r_{i 2} \mid r_{i 1}, \mathbf{x}_{i}, y_{i}, \xi_{2}\right)=\frac{\exp \left(r_{i 2} f_{i 2}\right)}{1+\exp \left(f_{i 2}\right)}, f_{i 1}=\xi_{10}+\xi_{11} x_{i 1}+\cdots+$ $\xi_{15} x_{i 5}+\xi_{16} y_{i}$ and $f_{i 2}=\xi_{20}+\xi_{21} x_{i 1}+\cdots+\xi_{25} x_{i 5}+\xi_{27} r_{i 1}$. Then, we considered a global perturbation

$$
\begin{aligned}
p\left(\mathbf{r}_{i} \mid \mathbf{x}_{i}, \mathbf{z}_{i}, y_{i}, \xi, \omega\right)= & p\left(r_{i 2} \mid r_{i 1}, \mathbf{x}_{i}, \mathbf{z}_{i}, y_{i}, \xi_{2}, \omega\right) \\
& \times p\left(r_{i 1} \mid \mathbf{x}_{i}, \mathbf{z}_{i}, y_{i}, \xi_{1}, \omega\right) \\
p\left(r_{i 1} \mid \mathbf{x}_{i}, \mathbf{z}_{i}, y_{i}, \xi_{1}, \omega\right)= & \frac{\exp \left[r_{i 1}\left(f_{i 1}+\omega_{1} z_{i 1}+\omega_{2} z_{i 2}\right)\right]}{1+\exp \left(f_{i 1}+\omega_{1} z_{i 1}+\omega_{2} z_{i 2}\right)}, \\
p\left(r_{i 2} \mid r_{i 1}, \mathbf{x}_{i}, \mathbf{z}_{i}, y_{i}, \xi_{2}, \omega\right)= & \frac{\exp \left[r_{i 2}\left(f_{i 2}+\omega_{3} z_{i 1}+\omega_{4} z_{i 2}\right)\right]}{1+\exp \left(f_{i 2}+\omega_{3} z_{i 1}+\omega_{4} z_{i 2}\right)} .
\end{aligned}
$$

The principal eigenvalue of $\mathrm{FI}_{f l r}\left(\boldsymbol{\omega}^{0}\right)$ was 0.11 , far smaller than the weighted chi-squared 0.05 cut-off point. This may suggest that the missing data mechanism is likely to be MAR.

In fitting the model using equation (25), the large value of the estimate for $\xi_{26}$ indicated that the missingness of $x_{2}$ might depend on the response, whereas the estimates for all other $\xi \mathrm{s}$ were nonsignificant. Thus, we dropped the $y_{i}$ term in $f_{i 2}$ of equation (25), leading to $f_{i 2}=$ $\xi_{20}+\xi_{21} x_{i 1}+\cdots+\xi_{25} x_{i 5}+\xi_{27} r_{i 1}$. Then we used the global perturbation in equation (26) with

$$
\begin{aligned}
p\left(r_{i 1} \mid \mathbf{x}_{i}, y_{i}, \xi_{1}, \omega\right) & =\frac{\exp \left(r_{i 1} f_{i 1}\right)}{1+\exp \left(f_{i 1}\right)} \text { and } \\
p\left(r_{i 2} \mid r_{i 1}, \mathbf{x}_{i}, y_{i}, \xi_{2}, \omega\right) & =\frac{\exp \left[r_{i 2}\left(f_{i 2}+\omega y_{i}\right)\right]}{1+\exp \left(f_{i 2}+\omega y_{i}\right)} .
\end{aligned}
$$

It turned out that $\mathrm{FI}_{f l r}\left(\omega^{0}\right)$ was 4.51 , which is larger than the chi-squared 0.05 cut-off point. This suggests that the missingness of $x_{2}$ may depend on the response.

Furthermore, to assess the linear relationship between the response and the covariates $\left(z_{1}, z_{2}\right)$, we employed a global perturbation scheme as follows:

$$
\begin{aligned}
y_{i}= & \beta_{0}+\beta_{1} z_{i 1}+\beta_{2} z_{i 2}+\beta_{3} x_{i 1}+\cdots+\beta_{7} x_{i 5} \\
& +\sum_{j=1}^{m+3} \omega_{j} B_{j}\left(z_{i 1}\right)+\sum_{j=1}^{m+3} \omega_{j+m+3} B_{j}\left(z_{i 2}\right)+\epsilon_{i},
\end{aligned}
$$

where the $B_{j}(z)$ are truncated polynomials of order 2 to 4 given by $z^{2}, z^{3}, z^{4},\left(z-k_{1}\right)_{+}^{4}, \ldots,\left(z-k_{m}\right)_{+}^{4}$, where $k_{1}, \ldots, k_{m}$ are the $m=3$ prefixed knots. The principal eigenvalue of $\mathrm{FI}_{f l r}(\boldsymbol{\omega})$ was 3.44, which was not statistically significant at the 5\% significance level $(p$-value $=0.65)$. Thus, the fitted model appears to be robust to this global perturbation scheme.

\subsection{Liver Cancer Data}

To further illustrate our proposed methods, we revisit the liver cancer data as introduced in Section 1 (Ibrahim, Chen, and Lipsitz, 1999). We are interested in how the number of cancerous liver nodes $(y)$ when entering the trials is predicted by six other baseline characteristics: time since diagnosis of the disease (in weeks) $\left(z_{1}\right)$; two biochemical markers (each classified as normal or abnormal), alpha-fetoprotein $\left(z_{2}\right)$ and anti-hepatitis B antigen $\left(z_{3}\right)$; associated jaundice (yes, no) $\left(x_{1}\right)$; body mass index (weight in kilograms divided by the square of height in meters) $\left(x_{2}\right)$; and age (in years) $\left(x_{3}\right)$. 
We used a Poisson regression model, $p\left(y_{i} \mid \mathbf{x}_{i}, \mathbf{z}_{i}, \beta\right) \propto \exp \left[y_{i}\left(\mathbf{v}_{i}^{T} \beta\right)-\exp \left(\mathbf{v}_{i}^{T} \beta\right)\right]$, where $\mathbf{v}_{i}^{T}=\left(1, x_{i 1}, x_{i 2}, x_{i 3}, z_{i 1}, z_{i 2}, z_{i 3}\right)$ is the $1 \times 7$ vector of covariates including an intercept, and $\boldsymbol{\beta}=$ $\left(\beta_{0}, \beta_{1}, \ldots, \beta_{6}\right)^{T}$ are the corresponding regression coefficients. Logarithm of the time since diagnosis was used to achieve approximate normality. Because only $\mathbf{z}_{i}=\left(z_{i 1}, z_{i 2}, z_{i 3}\right)$ has missing values, we need to consider a joint distribution only for these covariates. Because $z_{i 2}$ and $z_{i 3}$ were both dichotomous, we used logistic regressions. Thus,

$$
\begin{aligned}
p\left(z_{i 1}, z_{i 2}, z_{i 3} \mid \mathbf{x}_{i}, \alpha\right)= & p\left(z_{i 3} \mid z_{i 1}, z_{i 2}, \mathbf{x}_{i}, \alpha_{3}\right) \\
& \times p\left(z_{i 2} \mid z_{i 1}, \mathbf{x}_{i}, \alpha_{2}\right) \times p\left(z_{i 1} \mid \mathbf{x}_{i}, \alpha_{1}\right),
\end{aligned}
$$

where $\boldsymbol{\alpha}=\left(\boldsymbol{\alpha}_{1}, \boldsymbol{\alpha}_{2}, \boldsymbol{\alpha}_{3}\right)$ and $\left(z_{i 3} \mid z_{i 1}, z_{i 2}, \mathbf{x}_{i}\right)$ is a logistic regression with

$$
\begin{aligned}
p\left(z_{i 3}\right. & \left.=1 \mid z_{i 1}, z_{i 2}, \mathbf{x}_{i}, \alpha_{3}\right) \\
& =\frac{\exp \left(\alpha_{30}+\alpha_{31} z_{i 1}+\alpha_{32} z_{i 2}+\alpha_{3 x^{\prime}} \mathbf{x}_{i}\right)}{1+\exp \left(\alpha_{30}+\alpha_{31} z_{i 1}+\alpha_{32} z_{i 2}+\alpha_{3 x^{x}}^{T} \mathbf{x}_{i}\right.},
\end{aligned}
$$

and $\alpha_{3 x}^{T}=\left(\alpha_{33}, \alpha_{34}, \alpha_{35}\right)$. Similarly,

$$
p\left(z_{i 2}=1 \mid z_{i 1}, \mathbf{x}_{i}, \alpha_{2}\right)=\frac{\exp \left(\alpha_{20}+\alpha_{21} z_{i 1}+\alpha_{2 x}^{T} \mathbf{x}_{i}\right)}{1+\exp \left(\alpha_{20}+\alpha_{21} z_{i 1}+\alpha_{2 x}^{T} \mathbf{x}_{i}\right)},
$$

and $\alpha_{2 x}^{T}=\left(\alpha_{22}, \alpha_{23}, \alpha_{24}\right)$. In addition, we took a normal distribution for the missing covariate $z_{1}$, specifically, $z_{i 1} \sim N\left(\alpha_{11}, \alpha_{12}\right)$ and $\alpha_{1}^{T}=\left(\alpha_{11}, \alpha_{12}\right)$. We assumed that the missing covariates are MAR and estimated $(\boldsymbol{\beta}, \boldsymbol{\alpha})$ using the EM algorithm.

To detect the influential cases, we employed a perturbation to simultaneously perturb the missing covariates $z_{i 1}, z_{i 2}$, and $z_{i 3}$ such that $y_{i}=\beta_{0}+\beta_{1}\left(z_{i 1}+\omega_{i}\right)+\beta_{2}\left(z_{i 2}+\omega_{i}\right)+\beta_{3}\left(z_{i 3}+\omega_{i}\right)$ $+\cdots+\beta_{6} x_{i 3}+\epsilon_{i}$. Both $\mathrm{FI}_{\hat{\eta} o}\left(\omega^{0}\right), \mathbf{e}_{i}$ and $\mathrm{FI}_{f l r}\left(\omega^{0}\right), \mathbf{e}_{i}$ indicated that cases 10, 15, 65, and 160 were very influential for this perturbation (Figures $4 a$ and $b$ ). Then we employed a perturbation to the distribution of $z_{i 1}$ such that $z_{i 1} \sim N\left(\alpha_{11}+\omega_{i}, \alpha_{12}\right), i=1, \ldots, n$, and both influence measures detected case 131 to be influential for the distributional assumption of $z_{i 1}$ (Figures $4 \mathrm{c}$ and d). These findings confirmed the suspected cases reported in Table 1 .

Next, we examined the functional form of the missing data mechanism given by

$$
\begin{aligned}
p\left(\mathbf{r}_{i} \mid \mathbf{x}_{i}, y_{i}, \xi\right)= & p\left(r_{i 3} \mid r_{i 1}, r_{i 2}, \mathbf{x}_{i}, y_{i}, \xi_{2}\right) \\
& \times p\left(r_{i 2} \mid r_{i 1}, \mathbf{x}_{i}, y_{i}, \xi_{2}\right) \times p\left(r_{i 1} \mid \mathbf{x}_{i}, y_{i}, \xi_{1}\right), \\
p\left(r_{i 1} \mid y_{i}, \mathbf{x}_{i}, \xi_{1}\right)=\frac{\exp \left(r_{i 1} f_{i 1}\right)}{1+\exp \left(f_{i 1}\right)}, & \\
p\left(r_{i 2} \mid r_{i 1}, y_{i}, \mathbf{x}_{i}, \xi_{2}\right)= & \frac{\exp \left(r_{i 2} f_{i 2}\right)}{1+\exp \left(f_{i 2}\right)},
\end{aligned}
$$




$$
p\left(r_{i 3} \mid r_{i 1}, r_{i 2}, y_{i}, \mathbf{x}_{i}, \xi_{3}\right)=\frac{\exp \left(r_{i 3} f_{i 3}\right)}{1+\exp \left(f_{i 3}\right)},
$$

in which $f_{i 1}=\xi_{10}+\xi_{11} x_{i 1}+\xi_{12} x_{i 2}+\xi_{13} x_{i 3}+\xi_{14} y_{i}, f_{i 2}=\xi_{20}+\xi_{21} x_{i 1}+\xi_{22} x_{i 2}+\xi_{23} x_{i 3}+\xi_{24} y_{i}$ $+\xi_{25} r_{i 1}$, and $f_{i 3}=\xi_{30}+\xi_{31} x_{i 1}+\xi_{32} x_{i 2}+\xi_{33} x_{i 3}+\xi_{34} y_{i}+\xi_{35} r_{i 1}+\xi_{36} r_{i 2}$. Then, we considered a global perturbation for the missing mechanism:

$$
\begin{aligned}
p\left(\mathbf{r}_{i} \mid \mathbf{x}_{i}, \mathbf{z}_{i}, y_{i}, \xi, \omega\right)= & p\left(r_{i 3} \mid r_{i 1}, r_{i 2}, \mathbf{x}_{i}, \mathbf{z}_{i}, y_{i}, \xi_{2}, \omega\right) \\
& \times p\left(r_{i 2} \mid r_{i 1}, \mathbf{x}_{i}, \mathbf{z}_{i}, y_{i}, \xi_{2}, \omega\right) \\
& \times p\left(r_{i 1} \mid \mathbf{x}_{i}, \mathbf{z}_{i}, y_{i}, \xi_{1}, \omega\right)
\end{aligned}
$$

$$
\begin{aligned}
p & \left(r_{i 1} \mid \mathbf{x}_{i}, \mathbf{z}_{i}, y_{i}, \xi_{1}, \omega\right) \\
& =\frac{\exp \left[r_{i 1}\left(f_{i 1}+\omega_{1} z_{i 1}+\omega_{2} z_{i 2}+\omega_{3} z_{i 3}\right)\right]}{1+\exp \left(f_{i 1}+\omega_{1} x_{i 1}+\omega_{2} x_{i 2}+\omega_{3} z_{i 3}\right)}, \\
p( & \left(r_{i 2} \mid r_{i 1}, \mathbf{x}_{i}, \mathbf{z}_{i}, y_{i}, \xi_{2}, \omega\right) \\
& =\frac{\exp \left[r_{i 2}\left(f_{i 2}+\omega_{4} z_{i}+\omega_{5} z_{i 2}+\omega_{6} z_{i 3}\right)\right]}{1+\exp \left(f_{i 2}+\omega_{4} z_{i}+\omega_{5} z_{i 2}+\omega_{6} z_{i 3}\right)}, \\
p & \left(r_{i 3} \mid r_{i 2}, r_{i 1}, \mathbf{x}_{i}, \mathbf{z}_{i}, y_{i}, \xi_{2}, \omega\right) \\
& =\frac{\exp \left[r_{i 3}\left(f_{i 3}+\omega_{7} z_{i 1}+\omega_{8} z_{i 2}+\omega_{9} z_{i 3}\right)\right]}{1+\exp \left(f_{i 3}+\omega_{7} z_{i 1}+\omega_{8} z_{i 2}+\omega_{9} z_{i 3}\right)} .
\end{aligned}
$$

The principal eigenvalue of $\mathrm{FI}_{f l r}\left(\boldsymbol{\omega}^{0}\right)(0.24)$ was quite small, which suggests that the missing data mechanism is likely to be MAR. Following the arguments in Zhu et al. (2007), we considered a single-case perturbation for the missing mechanism as follows:

$$
\begin{aligned}
p & \left(r_{i 1} \mid \mathbf{x}_{i}, \mathbf{z}_{i}, y_{i}, \boldsymbol{\xi}_{1}, \omega\right) \\
& =\frac{\exp \left[r_{i 1}\left(f_{i 1}+\omega_{i}\left(z_{i 1} / s_{z 1}+z_{i 2} / s_{z 2}+z_{i 3} / s_{z 3}\right)\right)\right]}{1+\exp \left(f_{i 1}+\omega_{i}\left(z_{i 1} / s_{z 1}+z_{i 2} / s_{z 2}+z_{i 3} / s_{z 3}\right)\right)},
\end{aligned}
$$

where $s_{z 1}, s_{z 2}$, and $s_{z 3}$ are the sample standard deviations for $z_{1}, z_{2}$, and $z_{3}$, respectively. Then, a similar perturbation was introduced for $r_{i 2}$ and $r_{i 3}$. All perturbations revealed case 131 to be influential. However, the perturbation for $r_{i 3}$ revealed only case 65 as an influential case. The reason that cases 10,15 , and 160 did not stand out under the single-case perturbation for all missing covariates and case 65 did not stand out under the single-case perturbation for $z_{1}$ or $z_{2}$, is that: (i) they all have very large values in the response, (ii) large response values $y_{i}$ tend to yield large values of $p\left(r_{i}=1 \mid \mathbf{x}_{i}, y_{i}, \boldsymbol{\xi}\right)$ for all $z_{1}, z_{2}$, and $z_{3}$, and (iii) cases 10,15 , and 160 have no missing values in $z_{1}, z_{2}$, and $z_{3}$ so they fit equations (27), (28), and (29) well, whereas case 65 has no missing values in $z_{1}$ and $z_{2}$ so it fits equations (27) and (28) well.

\section{Discussion}

We have developed a general local influence methodology for carrying out sensitivity analyses in GLMs with MAR or NMAR covariate data. We have also proposed a novel methodology for choosing an appropriate perturbation scheme and examined several influence measures within this context. The simulation studies and the real datasets showed very promising results for the proposed methods. We emphasize again that in missing data problems, there is typically little information in the data regarding the form of the missing data mechanism, and the parametric assumption of the missing data mechanism itself is not "testable" from the data. Thus, NMAR modeling should be viewed as a sensitivity analysis concerning a more complicated model. In this sense, it is not advisable to carry out formal tests directly to assess and compare MAR and NMAR models. Future work in this area includes extending these 
methodologies to the Cox proportional hazards model with right censored survival data and missing covariates, as well as to parametric and semi-parametric models for longitudinal data with MAR or NMAR response and/or covariate data.

\section{Acknowledgments}

This work was supported in part by National Science Foundation (NSF) grants SES-06-43663 and BCS-08-26844 and National Institutes of Health (NIH) grants ULI-RR025747-01 and AG033387 to Dr Zhu and NIH grants GM70335 and CA74015 to Dr Ibrahim. We thank the editor and two referees for variable suggestions that greatly improved the article.

\section{References}

Beckman RJ, Nachtsheim CJ, Cook RD. Diagnostics for mixed-model analysis of variance. Technometrics 1987;29:413-426.

Cook RD. Assessment of local influence (with discussion). Journal of the Royal Statistical Society, Series B 1986;48:133-169.

Copas JB, Eguchi S. Local sensitivity approximations for selectivity bias. Journal of the Royal Statistical Society, Series B 2001;63:871-895.

Copas JB, Eguchi S. Local model uncertainty and incomplete-data bias (with discussion). Journal of the Royal Statistical Society, Series B 2005;67:459-513.

Copas JB, Li HG. Inference for non-random samples (with discussion). Journal of the Royal Statistical Society, Series B 1997;59:55-96.

Cox DR, Reid N. Parameter orthogonality and approximate conditional inference (with discussion). Journal of the Royal Statistical Society, Series B 1987;49:1-39.

Gustafson P. On measuring sensitivity to parametric model misspecification. Journal of the Royal Statistical Society, Series B 2001;63:81-94.

Hens N, Aerts, Molenberghs G, Thijs H, Verbeke G. Kernel weighted influence measures. Computational Statistics and Data Analysis 2005;48:467-487.

Ibrahim JG, Chen MH, Lipsitz SR. Monte Carlo EM for missing covariates in parametric regression models. Biometrics 1999;55:591-596. [PubMed: 11318219]

Ibrahim JG, Lipsitz SR, Chen MH. Missing covariates in generalized linear models when the missing data mechanism is nonignorable. Journal of the Royal Statistical Society, Series B 1999;61:173-190.

Jansen I, Molenberghs G, Aerts M, Thijs H, Van Steen K. A local influence approach to binary data from a psychiatric study. Biometrics 2003;59:410-419. [PubMed: 12926726]

Jansen I, Hens N, Molenberghs G, Aerts M, Verbeke G, Kenward MG. The nature of sensitivity in monotone missing not at random models. Computational Statistics and Data Analysis 2006;50:830858.

Lipsitz SR, Ibrahim JG. A conditional model for incomplete covariates in parametric regression models. Biometrika 1996;83:916-922.

Thomas W, Cook RD. Assessing influence on regression coefficients in generalized linear models. Biometrika 1989;76:741-749.

Troxel AB. A comparative analysis of quality of life data from a southwest oncology group randomized trial of advanced colorectal cancer. Statistics in Medicine 1998;17:767-779. [PubMed: 9549822]

Troxel AB, Ma G, Heitjan DF. An index of local sensitivity to nonignorability. Statistica Sinica 2004;14:1221-1237.

Van Steen K, Molenberghs G, Thijs H. A local influence approach to sensitivity analysis of incomplete longitudinal ordinal data. Statistical Modelling: An International Journal 2001;1:125-142.

Verbeke G, Molenberghs G, Thijs H, Lasaffre E, Kenward MG. Sensitivity analysis for non-random dropout: A local influence approach. Biometrics 2001;57:43-50. [PubMed: 11252617]

Zhu HT, Lee SY. Local influence for incomplete-data models. Journal of the Royal Statistical Society, Series B 2001;63:111-126.

Zhu HT, Ibrahim JG, Lee S, Zhang HP. Assessment of local influence by invariant measures. Annals of Statistics 2007;35:2565-2588. 


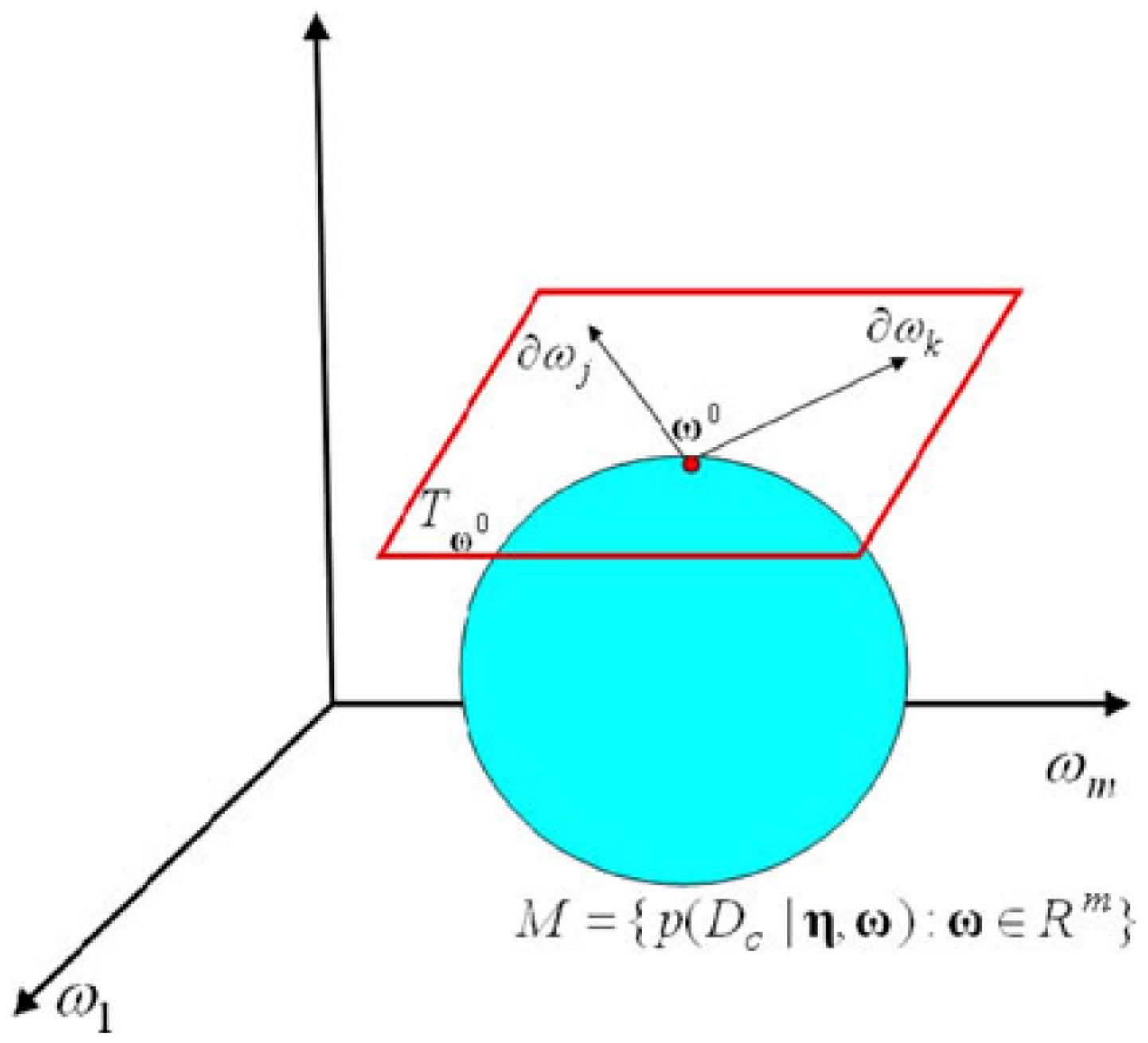

Figure 1.

A graphical representation of the perturbation manifold. 

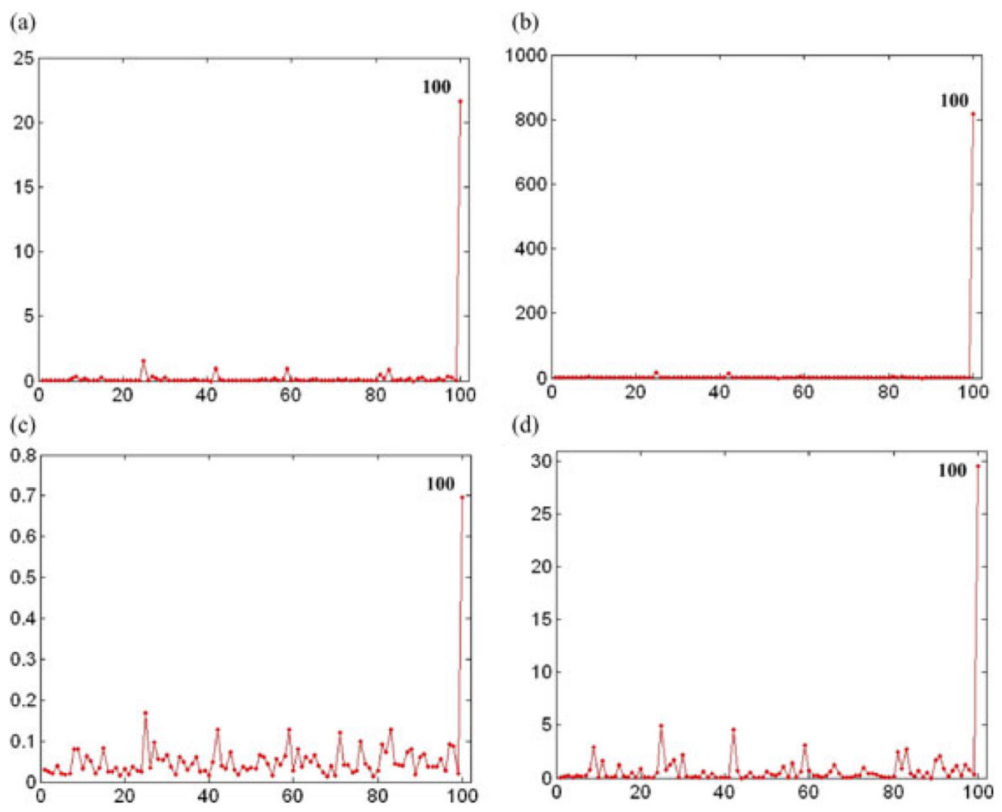

Figure 2.

Index plots of influence measures from a simulated dataset with $y_{100}$ as an influential case: (a) $\mathrm{FI}_{\boldsymbol{\eta}^{\wedge} o\left(\omega^{0}\right), \mathbf{e}_{i}}$ and (b) $\mathrm{FI}_{f l r}\left(\boldsymbol{\omega}^{0}\right), \mathbf{e}_{i}$ for the variance perturbation; (c) $\mathrm{FI}_{\boldsymbol{\eta}^{\wedge} o}\left(\omega^{0}\right), \mathbf{e}_{i}$ and (d) $\mathrm{FI}_{f l r}\left(\omega^{0}\right), \mathbf{e}_{i}$ for the missing covariate perturbation. 
(a)

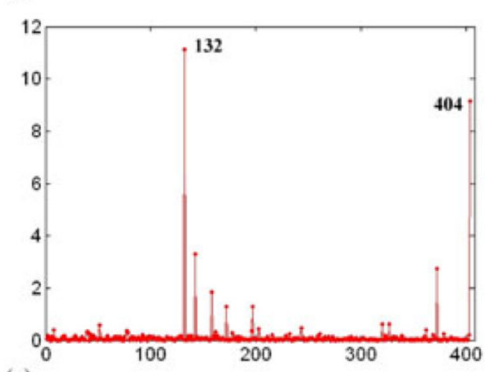

(c)

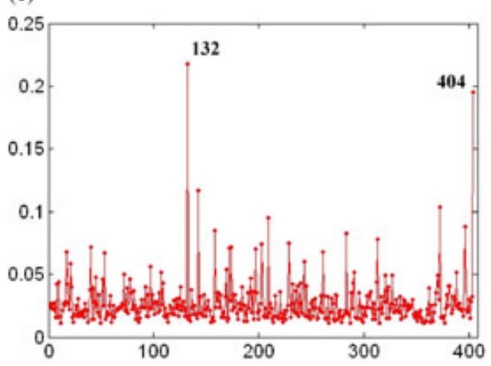

(b)

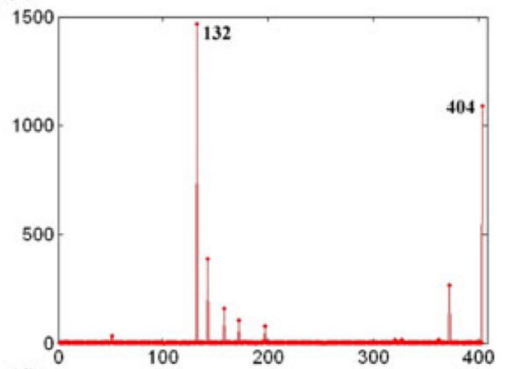

(d)

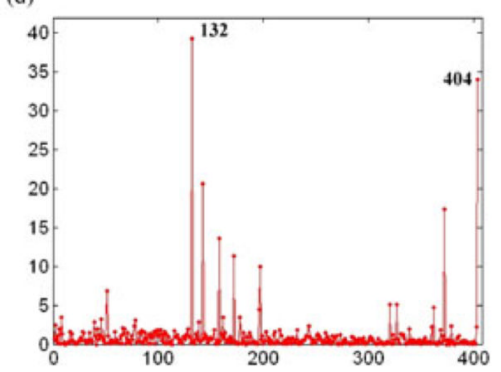

Figure 3.

Index plots of influence measures for quality-of-life data: (a) $\mathrm{FI}_{\hat{\eta} o}\left(\omega^{0}\right), \mathbf{e}_{i}$ and (b) $\mathrm{FI}_{f l r}$ $\left(\omega^{0}\right), \mathbf{e}_{i}$ for the variance perturbation; (c) $\mathrm{FI}_{\hat{\eta} o}\left(\omega^{0}\right), \mathbf{e}_{i}$ and (d) $\mathrm{FI}_{f l r}\left(\boldsymbol{\omega}^{0}\right), \mathbf{e}_{i}$ for the missing covariate perturbation. 
(a)

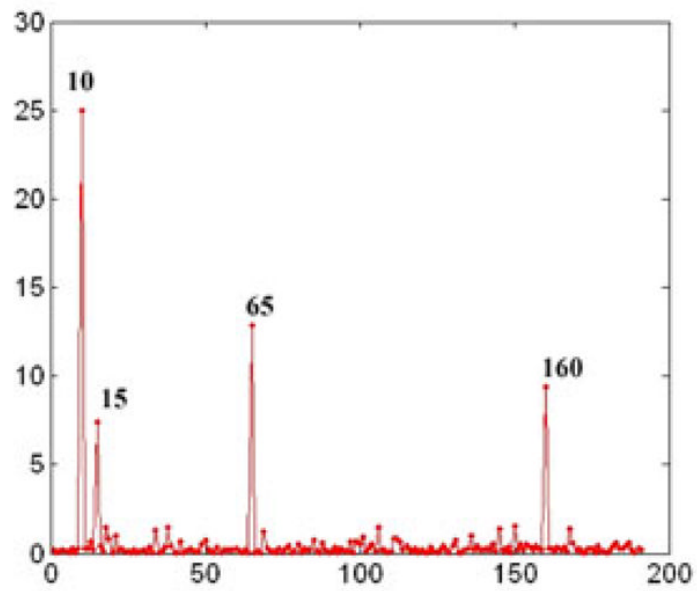

(c)

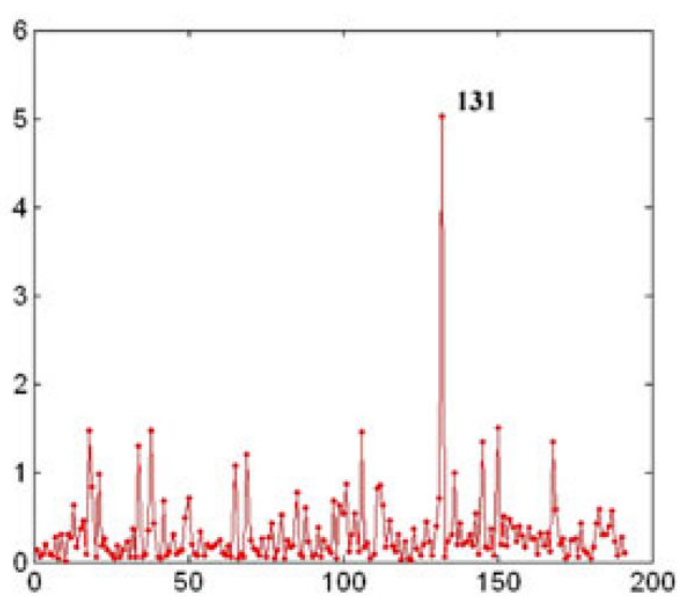

(b)

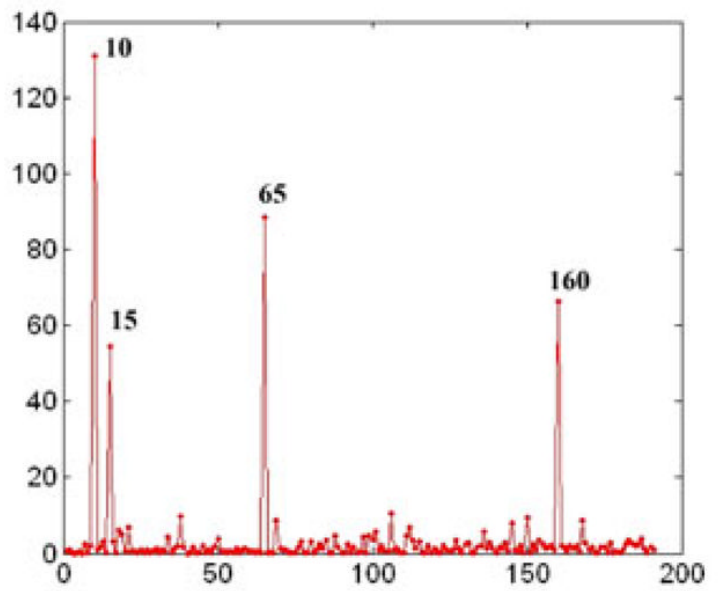

(d)

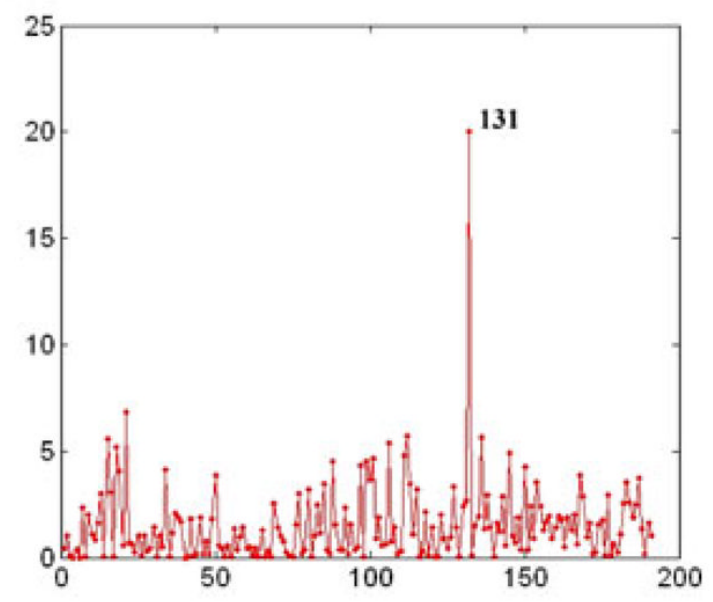

Figure 4.

Index plots of influence measures for liver cancer data: (a) $\mathrm{FI}_{\hat{\eta} o}\left(\omega^{0}\right), \mathbf{e}_{i}$ and (b) $\mathrm{FI}_{f l r}\left(\omega^{0}\right), \mathbf{e}_{i}$ for the missing covariate perturbation; (c) $\mathrm{FI}_{\hat{\eta} o}\left(\omega^{0}\right), \mathbf{e}_{i}$ and (d) $\mathrm{FI}_{f l r}\left(\omega^{0}\right), \mathbf{e}_{i}$ for the perturbation to the distribution of $z_{i 1}$. 


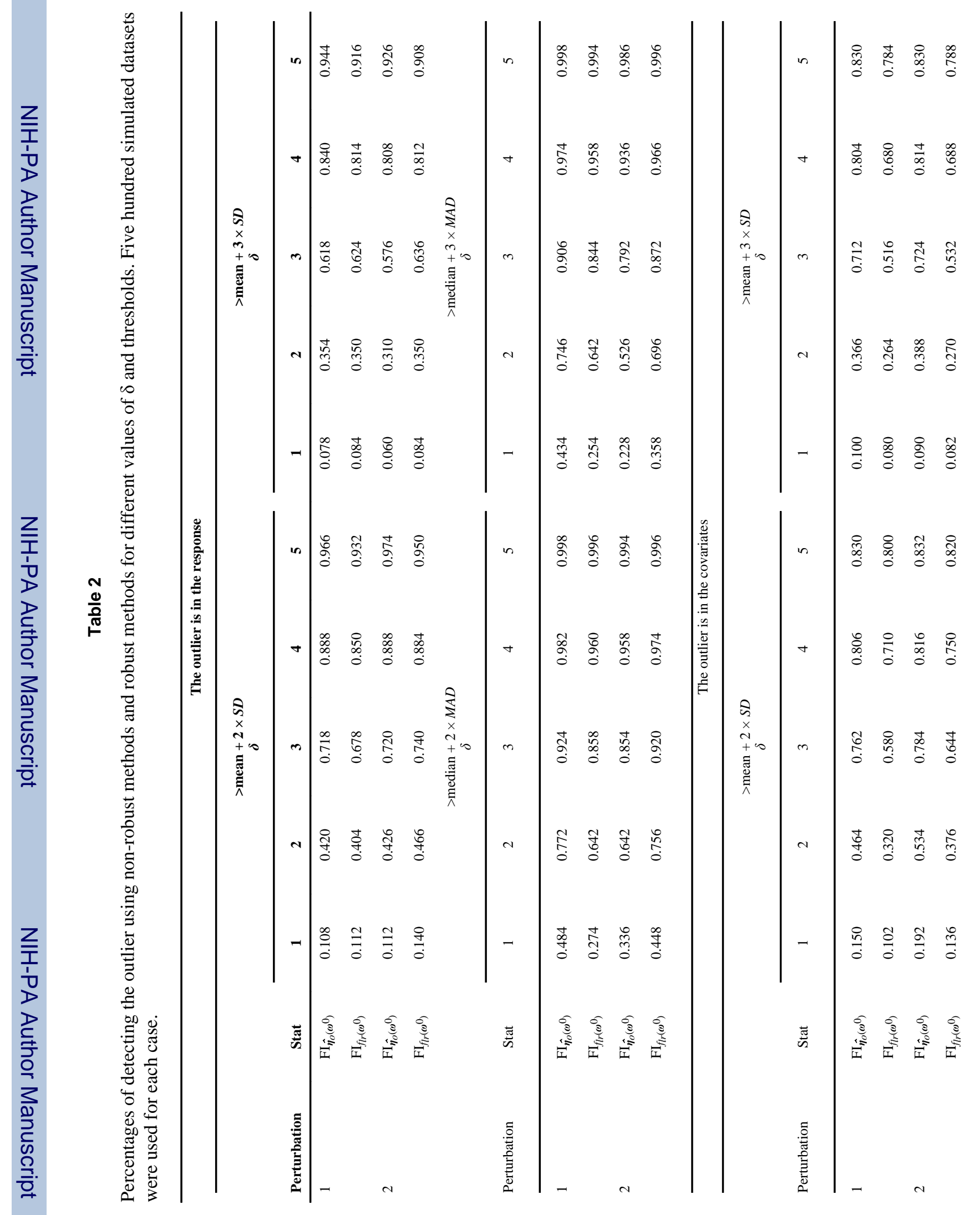


Shi et al.

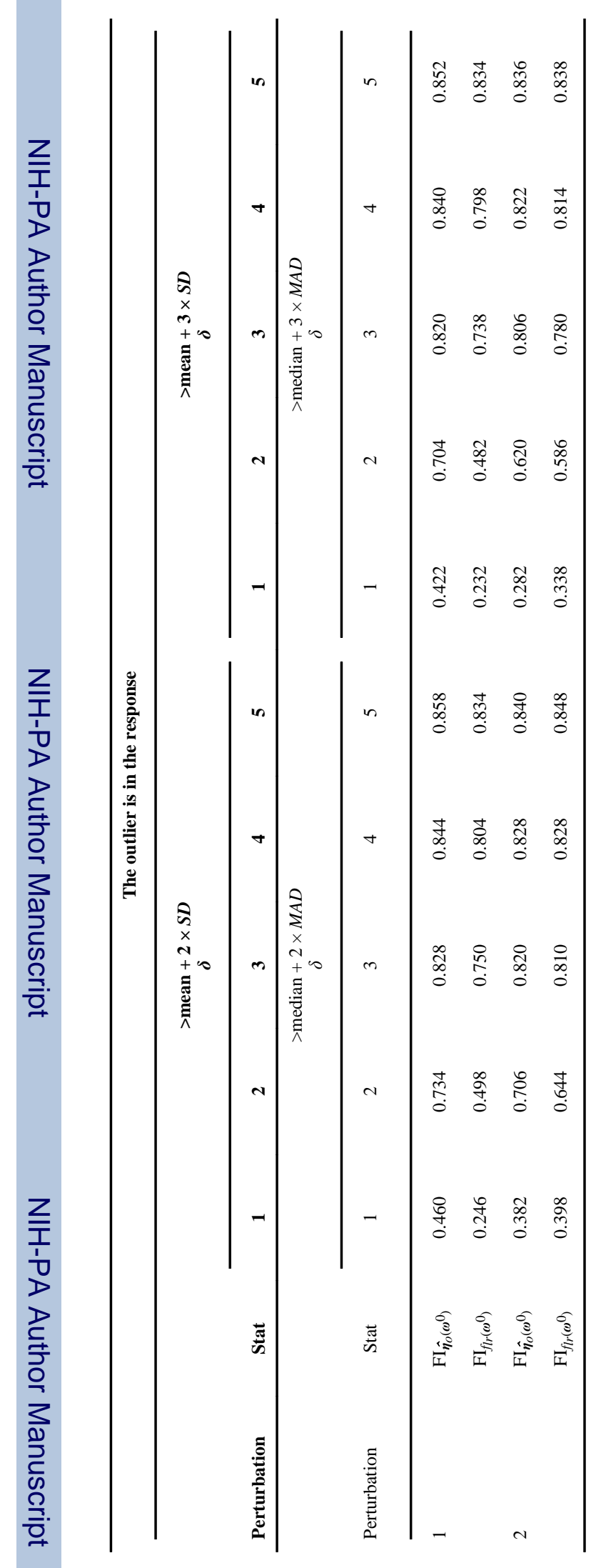

Biometrics. Author manuscript; available in PMC 2010 February 10. 